\title{
A Proposed Machine Learning Based Collective Disease Model to Enable Predictive Diagnostics in Necrotising Enterocolitis
}

\author{
Jacqueline van Druten \\ School of Architecture, Computing and Engineering, \\ College of Arts, Technology and Innovation, UEL. \\ University Way, Dockland Campus \\ London, E16 2RD, UK. \\ u1639256@uel.ac.uk \\ Minesh Khashu \\ Neonatal unit, Poole Hospital NHS Foundation Trust \\ BH15 2JB, UK \\ Perinatal Health, Bournemouth University, UK.
}

\author{
Mhd Saeed Sharif \\ School of Architecture, Computing and Engineering, \\ College of Arts, Technology and Innovation, UEL. \\ University Way, Dockland Campus \\ London, E16 2RD, UK \\ s.sharif@uel.ac.uk \\ Hassan Abdalla \\ School of Architecture, Computing and Engineering \\ College of Arts, Technology and Innovation, UEL. \\ University Way, Dockland Campus \\ London, E16 2RD, UK
}

\begin{abstract}
Despite 60 years of research into necrotising enterocolitis (NEC), our understanding of the disease has not improved enough to achieve better outcomes. Even though NEC has remained the leading cause of death and poor outcomes in preterm infants, there remain vital questions on how to define, differentiate and detect the condition. Numerous international groups have recently highlighted NEC as a research priority and called for broader engagement of the scientific community to move the field forward. The three foremost barriers at present are lack of suitable definition(s), lack of clean datasets and consequently a lack of scope to gain sufficient insights from data. This research paper proposes a new direction of travel to advance neonatal gastro-intestinal monitoring and strengthen our efforts to gain better insights from global databases. An integrated machine learning based model is recommended to produce a comprehensive disease model to manage the complexity of this multi-variate disease. This intelligent disease model would be used in the daily neonatal settings to help aggregate data to support clinical decision making, better capture the complexity of each patient to enrich global datasets to create bigger and better data. This paper reviews current machine learning and CAD technologies in neonatology and suggests an innovative approach for an NEC disease model.
\end{abstract}

Keywords- Machine Learning, Necrotising Enterocolitis, NEC, Big Data, AI Based Diagnosis, Predictive Modelling, Multi-Variate Analysis, Deep Learning, Ensemble Modelling, Computer Assisted Detection, Disease Model.

\section{INTRODUCTION: THE CHALLENGE OF NEC}

Necrotizing enterocolitis (NEC) is considered the most devastating gastrointestinal emergency in the neonatal unit. It has remained a significant cause of neonatal death and poor developmental outcomes with a mortality of up to $60 \%$ [1]. Extensive research has been performed to understand NEC better, but despite these efforts, it remains an elusive disease to diagnose and differentiate from other Acquired Neonatal Intestinal Diseases (ANID) [2].

Acquired Neonatal Intestinal Diseases (ANID) is a group of conditions that are complex multi-variate conditions that are difficult to delineate, detect and diagnose. Of concern, is the disease course of ANID conditions such as SIP and NEC as they can be unpredictable and can rapidly progress to peritonitis and shock. Highlighting the urgent need for noninvasive, readily available and dynamic computer-aided detection support in clinical practice. ANID research also lacks a structure to uniformly capture the complexities of a patient's presentation in its entirety to recreate rich data sets that can globally be aggregated to create big data for research.

Timely diagnosis and monitoring is an important focus area for current NEC research as it can improve outcomes [2]. In current practice, the diagnosis of SIP and NEC is made based on clinical findings, laboratory investigations and medical imaging. The diagnosis strongly relies on imaging, as it provides an objective measurement to move beyond suspected NEC (suggestive clinical findings) to medically confirm NEC. However, imaging interpretation can also be challenging showing great inter-expert variability, as nonspecific results do not preclude NEC entirely [3]. There is currently no computer-aided detection software (CAD) for abdominal radiographs (AR) or abdominal ultrasound (AUS) in neonates. Newer imaging modalities such as AUS has excellent potential to outperform AR but unfortunately is underutilised. The use of AUS has been mainly limited to when AR findings are inconclusive. This is despite AUS showing several advantages over AR and outperforming AR in diagnosing NEC [4].

It is unlikely that traditional clinical research methods will progress NEC research as rapidly as machine learning (ML) and CAD enabled technology can. Although a randomised control trial is still considered the golden standard for univariate research to determine causality, observational trials as a research methodology have received renewed interest due to advanced analytic technologies being powerful enough for multivariable processing and management of confounders. Using real-world evidence (RWE), neonatology can overcome uncertainties about benefits, risk, real-life utilisation and performance of biomarkers or new therapies [5]. Obstetrics and Neonatology have limited ethical scope in trials approval, further advocating for insights from databases. ML can enable richer extraction of data, overcome bias (as it offers more objective 
processing) and lead to the extraction of better insights [6]. Furthermore, it is also able to cleanse existing databases of non-NEC entities, therefore improving the quality of current databases.

\section{Gaining Insights through Predictive Modeling OF CLINICAL PRESENTATION}

\section{A. Differentiating NEC from other ANID's clinical features}

The most common three forms of the Acquired neonatal intestinal disease (ANID) is: spontaneous intestinal perforations (SIP), classic necrotising enterocolitis (Preterm NEC) and Term-NEC (primarily seen in term infants) [7]. See Table I for their differential features.

TABLE I. DIFFERENTIAL FEATURES FOR THREE COMMON FORMS OF ANID [7].

\begin{tabular}{|c|c|c|}
\hline $\begin{array}{c}\text { Spontaneous } \\
\text { Intestinal Perforation }\end{array}$ & Preterm NEC & Term NEC \\
\hline $\begin{array}{l}\text { Onset of symptoms: } \\
\text { Birth weight }<1250 \mathrm{~g} \text { : } \\
\text { onset within first seven } \\
\text { days. } \\
\text { Birth weight }>1250 \mathrm{~g} \text { : } \\
\text { onset within two } \\
\text { weeks. }\end{array}$ & $\begin{array}{c}\text { Onset of symptoms: } \\
\text { Onset usually after two } \\
\text { weeks. } \\
\text { In cases that are } \\
\text { ischemia-driven, onset } \\
\text { is within first month of } \\
\text { life. }\end{array}$ & $\begin{array}{l}\text { Onset of symptoms: } \\
\text { Onset within the first } \\
\text { month of life, often } \\
\text { within } 11-25 \text { days. }\end{array}$ \\
\hline $\begin{array}{c}\text { Gestational age: } \\
\text { Most cases of SIP } \\
\text { involve infants }<30 \\
\text { weeks gestation and } \\
<1500 \mathrm{~g} . \\
\text { Older infants often } \\
\text { have IUGR }\end{array}$ & $\begin{array}{c}\text { Gestational age: } \\
<36 \text { weeks gestation }\end{array}$ & $\begin{array}{c}\text { Gestational age: } \\
\geq 36 \text { weeks gestation }\end{array}$ \\
\hline $\begin{array}{l}\text { Clinical Features: } \\
\text { Insidious onset of } \\
\text { symptoms. } \\
\text { In most cases affects } \\
\text { low birth weight } \\
(<1500 \mathrm{~g}) \text { with }<30 \\
\text { weeks gestation. } \\
\text { Abdominal distention } \\
\text { Blue-grey } \\
\text { discolouration of the } \\
\text { abdominal wall. } \\
\text { No enteral feeding } \\
\text { association. } \\
\text {. }\end{array}$ & $\begin{array}{c}\text { Clinical Features: } \\
\text { Abdominal distention, } \\
\text { ileus and/or blood in } \\
\text { stools. } \\
\text { Persistent platelet } \\
\text { consumption } \\
\text { Meets "Two out of } \\
\text { Three" rule* } \\
\text { Requires three } \\
\text { preconditions: } \\
\text { A. Naive bowel, food } \\
\text { in the bowel. } \\
\text { B. Trigger event. } \\
\text { C. Fits Sartwell's } \\
\text { model of disease onset. }\end{array}$ & $\begin{array}{c}\text { Clinical Features: } \\
\text { Abdominal distention, } \\
\text { ileus and/or bloody } \\
\text { stools. } \\
\text { Birth weight greater } \\
\text { than } 2500 \mathrm{~g} \text {. } \\
\text { Associated maternal } \\
\text { illicit drug use, } \\
\text { intestinal anomalies } \\
\text { and congenital heart } \\
\text { disease. }\end{array}$ \\
\hline $\begin{array}{c}\text { Imaging Features: } \\
\text { AUS or AR } \\
\text { visualisation of } \\
\text { Pneumoperitoneum } \\
\text { without pneumatosis. }\end{array}$ & $\begin{array}{c}\text { Imaging Features: } \\
\text { AUS or AR } \\
\text { visualisation of } \\
\text { Portal Venous Gas } \\
\text { (PVG) or Pneumatosis } \\
\text { Intestinalis (PI). }\end{array}$ & $\begin{array}{c}\text { Imaging Features: } \\
\text { AUS or AR } \\
\text { visualisation of } \\
\text { Portal Venous Gas } \\
\text { (PVG) or Pneumatosis } \\
\text { Intestinalis (PI). }\end{array}$ \\
\hline
\end{tabular}

\section{B. The rationale for CAD insights to improve clinical decision making.}

Clinicians are faced with ever-increasing demands on their time and expected to evaluate information across systems to extract insights to provide recommendations continually and quickly throughout the day. This requires the ongoing data collection and aggregation to assess the infant. Various cognitive shortcuts can develop, unknowingly. These paradigms are timesaving strategies, to enable the clinician to meet an ever-increasing workload.
Unfortunately, as various cognitive biases develop, this can result in clinicians making assumptions that are often only seen in retrospect.

By enabling CAD, it can work as the clinicians assistant in aggregating data and summarise findings. This can save a significant amount of time, free cognitive processing capacity to make more thorough enquiry of the summarised data and utilise more energy on patient care planning. Furthermore, as CAD does not rely on biased experience and didactics, more productive and more uniform data is created that can readily be aggregated to international databases.

\section{Predictive modelling trials relevant to $N E C$}

Predictive modelling of NEC's clinical features would utilise data from, but is not limited to, clinical findings, assessment scores, lab results and clinical outcomes (see Figure 1). It needs to be appreciated that some data varies from static (binary) to dynamic values (example respiratory status). The current guidance case definitions and differential features (as provided in Table I) should form the foundation to formulate the supervised machine learning model. Collaboration with systems researchers, clinicians and radiologists would be needed ongoing insight and final approval of results. Techniques in building a supervised model are very diverse in the literature and would require ongoing comparative assessments against standardised techniques versus an ensemble model to accomplish the highest area under the curve (AUC).

FIGURE 1: OVERVIEW OF SUITABLE NEC DATA SOURCES AND VARIOUS MACHINE LEARNING TECHNIQUES SUITABLE TO BUILD A PREDICTION MODEL.

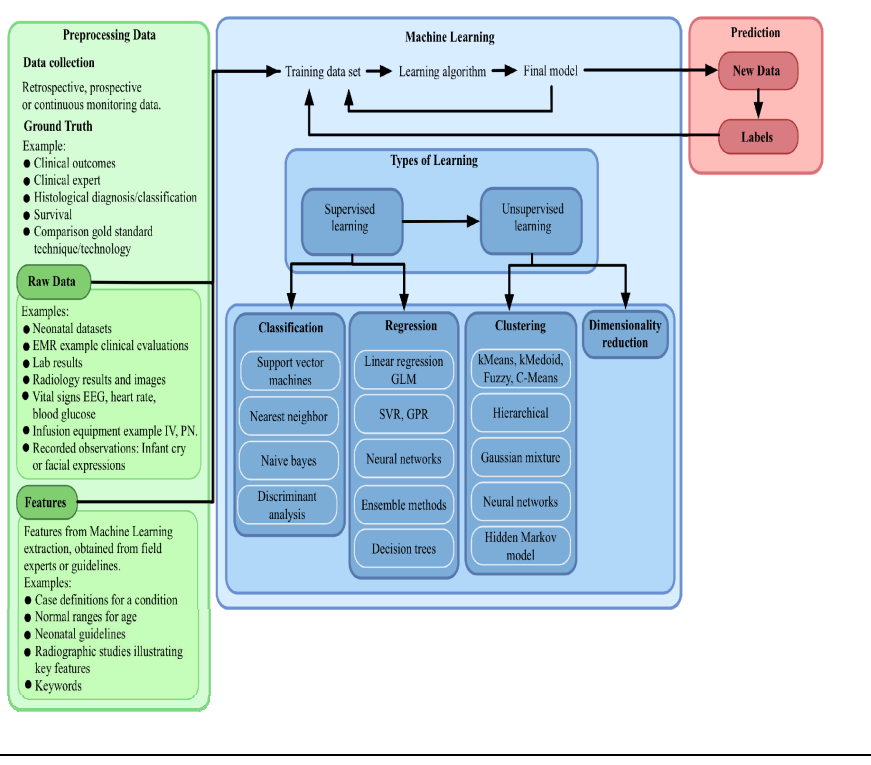

Figure 1 shows several such examples of how modelling could be approached. Of note these trials do deploy various techniques simultaneously of supervised and unsupervised models, again showing the heterogeneous nature of modelling. These are, but are not limited to, Support Vector Regressor (SVR), Support Vector Machines (SVM); Gaussian Processes Regression (GPR); Generalized linear model GLM and Fuzzy c-means (FCM). In the next section focus will be to elaborate on ML and Deep Learning trials in 
NEC or those that show promise in building a collective disease model (Figure 2).

Information can be extracted from neonatal electronic medical records (EMR) using classification models such as Support Vector machines and Naives Bayes. A recent systematic review looking into ML classification techniques [8], recommended extensive tuning of classification parameters. Recommending further refining of the impact of kernel type, adding or removing Gaussian mutation, gamma value, epsilon value, participant numbers, and C in SMV. In the case of Naïve Bayes classification, the smoothing values and baseline class weighing needed careful consideration to refine. Cohen et al. 2016, in his HER extraction only optimised only kernel type, $\mathrm{C}$, and gamma to improve accuracy [9]. His study also highlighted that careful consideration should be given to training database size, feature selection, classification technique, class balance, and reference baseline as they all significantly affected classification performance. In his trial, SMV outperformed Naives Bayes by AUC of 0.82 versus 0.71 .

Investigations into biomarkers in NEC have found that pairing clinical features with biomarkers can significantly improve predictive value. Classifying each case on clinical findings and validated biomarkers using Linear Discriminant Analysis (LDA) in an ensemble model, Sylvester et al. 2014, estimated the predictive value of three urine peptide biomarkers. He found modelling biomarker panel alone yielded an AUC of 0.858 . Clinical parameters alone had an AUC of 0.817 and left $40.1 \%$ of all patients in an 'indeterminate' risk group. Once three UPD parameters (fibrinogen peptides: FGA1883, FGA1826 and FGA2659) where added to the clinical model the AUC increased as high as 0.856. Illustrating a popular approach in biomarker research, to improve accuracy by incorporating a combination of various biomarkers and clinical findings. Reisinger et al, also studying biomarkers in NEC, found improved diagnostic accuracy of combining serum amyloid $\mathrm{A}$ and faecal calprotectin with intestinal fatty acid-binding protein. Accuracy improved when both faecal calprotectin and I-FABP where combined (sensitivity 94\%, specificity $79 \%$ ). As various biomarkers [10] are being investigated for various outcomes in NEC it would be advisable to create more sophisticated ensemble models to combine clinical and biomarker data to ease the interpretation for clinical practice.

Ensemble modelling can also test if prognostic variables are associated with a condition. This is especially useful in NEC as new biomarker combination, and imaging modalities (aka features) are being explored. Habibi et al. 2016 compared the use of Artificial Neural Networks (ANN MLP) versus Linear regression (LR) in ensemble models, in extracting prognostic factors and diagnose shunt infections using clinical information. ANN outperformed LR models in predicting shunt infection with an accuracy of $83.1 \%$ (AUC; 91.98\%, 95\% CI and $\mathrm{p}<0.001$ ) and 55.7\% (AUC; 76.5, 95\% CI). ANN showed five prognostic features including gestational age, weight, first shunt actual age, shunt revision, and myelomeningocele was significantly associated with shunt infection [11].

The predictive modelling needs for the clinical parameters of NEC would need a more dynamic and intuitive model. There are currently various assessment tools that have been developed to aid in the diagnosis of NEC [2], [12], [13] but these need to be comparatively tested in various settings to give a fair assessment of their performance. This would be useful to integrate into an ensemble model. An excellent example of such a model is the intelligent Jaundice diagnosis tool [21]. Hao et al. 2017, created an ensemble 'Jaundice' model using dynamic uncertain causality graph "chaining" inference algorithm and weighted logic operational flow for diagnostic reasoning. His model was able to integrate a diverse set of clinical features and their cut-off points. 'Jaundice' model achieved an accuracy of $99.01 \%$ and $84.73 \%$. The results had a better pattern of logic and were easier to interpret than conventional methods such as Bayesian Networks, providing more objectivity. This illustrates the potential use of a more sophisticated system suitable for NEC diagnosis.

Another sophisticated model is the three-dimensional mathematical and computational model of necrotising enterocolitis by Barber et al. 2013 [15]. This model shows great promise in advancing insights into pathophysiology. Unlike other ensemble models based on ordinary differential equations, their mathematic model considered both transient and spatial effects. Using cell-focussed fixed characteristics and a definitive Euler method, a structure of nonlinear transitionary sectional differential equations can be solved mathematically. The system monitors the evolution of an initial event (trauma) spatially in the intestinal wall. This model can provide an alternative or adjunct to animal trials to better explain the pathophysiological cascade of events. This will be particularly important when testing therapies and delineating various types of ANID.

\section{GAINING INSIGHTS THROUGH STANDARDISED CAD IMAGING AND PREDICTIVE MODELING OF FEATURES}

The timely diagnosis and monitoring is an important focus area for current research as it can improve outcomes in NEC [2]. In current practice, the diagnostic differentiation of SIP and NEC is based on clinical presentation, laboratory tests and imaging. The diagnosis of NEC strongly relies on imaging, as it provides an objective measurement to move beyond suspected NEC (suggestive clinical findings) to medically confirmed NEC, imaging of Portal Venous Gas (PVG) or Pneumatosis Intestinalis (PI).

Abdominal radiographs were the standard gold imaging for visualising pathognomonic signs of NEC. Recently, two new non-invasive imaging modalities have shown promise in NEC, Abdominal ultra-sound (AUS) and broadband optical spectroscopy (BOS). The challenge in neonatology is great inter-expert variability in interpreting findings. This is mainly in AUS as it is a newer modality without guidelines in NEC. CAD can be a crucial enabler to overcome these challenges in adapting newer non-invasive imaging modalities. Since 1960 's CAD has contributed significantly in AUS diagnostics. The first AUS CAD system was applied in the diagnosis of breast tumours [16]. CAD demonstrated improved the accuracy of diagnosis, reduced the time consumption, and decreases workload doctors [17]. Not surprisingly as the system enables guided assessment, rapid data processing, predictive analytic insights and enabled peer consensus from the clinician and radiologists [18].

\section{A. Challenges and opportunities for AUS}

The challenge in using AUS in ML is acquiring a standard plane (for feature extraction and classification), to then in turn define the diagnostic plane (for evaluation of 
changes and detection of pathognomonic findings). With radiology fellows, sonographers and clinicians this technique needs an experienced examiner. This very time-consuming even for experienced examiners and challenging for training fellows to master. Because the use of AUS in ANID (NEC) is new; finding experienced examiners is challenging, and with no standard guidelines we can expect the quality of insights to vary. Hence CAD has a critical role in integrating AUS in clinical practice, which in turn generates real-world data for validation of AUS in diagnostic criteria for various ANID's [19].

The benefits of deep learning (DL) is it can detect both a ultrasound standard plane and sonographic diagnostic plane automatically. The sonographic diagnostic plane can enable the detection of epithelial changes (thickening, thinning or perforation) and detect anomalies. Yaqub et al. 2017, showed that $\mathrm{CNN}$ can automatically detect a foetal neurosonographic diagnostic plane [20]. If the traditional method of feature input is used, then NEC AUS features as described by Faingold et al.2005 [21], Epelman et al. 2007 [22], Muchantef et al. 2013 [23], Bohnhorst et al. 2013 [24], Cuna et al. 2018 [25], [41], [42] should be considered to define the full breadth of textural and morphologic features.

Techniques for feature input or extraction of AUS differ between organ systems needing examination by their respective conditions. This also shows considerable variability in accuracy between organ systems rather than traditional versus DL techniques. This suggests that in developing an NEC AUS CAD, both traditional and DL systems need to be comparatively analysed to find the best accuracy. Textural features are the most commonly used for traditional feature input and are often combined with morphologic features. The classifying techniques often combined with supervised models are: SVM, ANN, FCN, decision trees and AdaBoost.

Recently, Ni et al. 2014, successfully developed an ML technique to acquire a standard plane in AUS in infants. In his study, he used a hierarchical, supervised learning framework to determine the standard plane from successive two-dimensional ultrasound images. The stomach bubble, umbilical vein and spine formed the critical anatomical coordinates for the standard plane in the ultrasound video. A radial component-based model was used to describe the spatial limits of these anatomical coordinates and novel discriminatory search approach too advantage of the vessel probability algorithm to estimate the likelihood of the vicinity of the spine and umbilical vein. Results from 223 new-born abdominal videos show it significantly outperformed total abdomen and other anatomy tracking software without spatial restrictions; the overall detection accuracy was $85.6 \%$ [28].

These results are at the lower range in comparison to other AUS CAD traditional techniques and DL. Hence it would be worth doing comparative trials using DL techniques such as GoogLeNet [29], [30], Boltzmann machine [31], Convolutional layers (CNN) [32], [33], Sparse encoder [34] and stacked denoising autoencoder (SDAE) [35] using his AUS standard plane from three key anatomical structures. To optimise accuracy other DL methods can be comparatively tested, for example looking at results from thyroid ultrasound models, accuracy was as high as 98.86$99.13 \%$ for GoogLeNet [29] and CNN [32] respectively. Whilst using traditional textural feature input with classification techniques using either SVM [36], FCN [37], decision tree's [38], ANN [39], or AdaBoost [40], we may see accuracy improve to 88.8 - 93,3 - 94,3-98, and $100 \%$ respectively.

\section{ClinicAl AND IMAgING PREDiCtive MODEL FOR NEC}

\section{A. Combined clinical and predictive imaging model}

Figure 2 shows combined traditional CAD and deep learning models for feature extraction and classification. Majority voting is used to choose the highest accuracy from the best combination of features and ML techniques. Once the best model for each of the sections, clinical and, is determined this will form the collective disease model by combination to try and further improve the accuracy of the case definition and find differential features to build each entity of ANIDS.

Figure 2: Proposed Collective disease MOdEL FOR NEC.

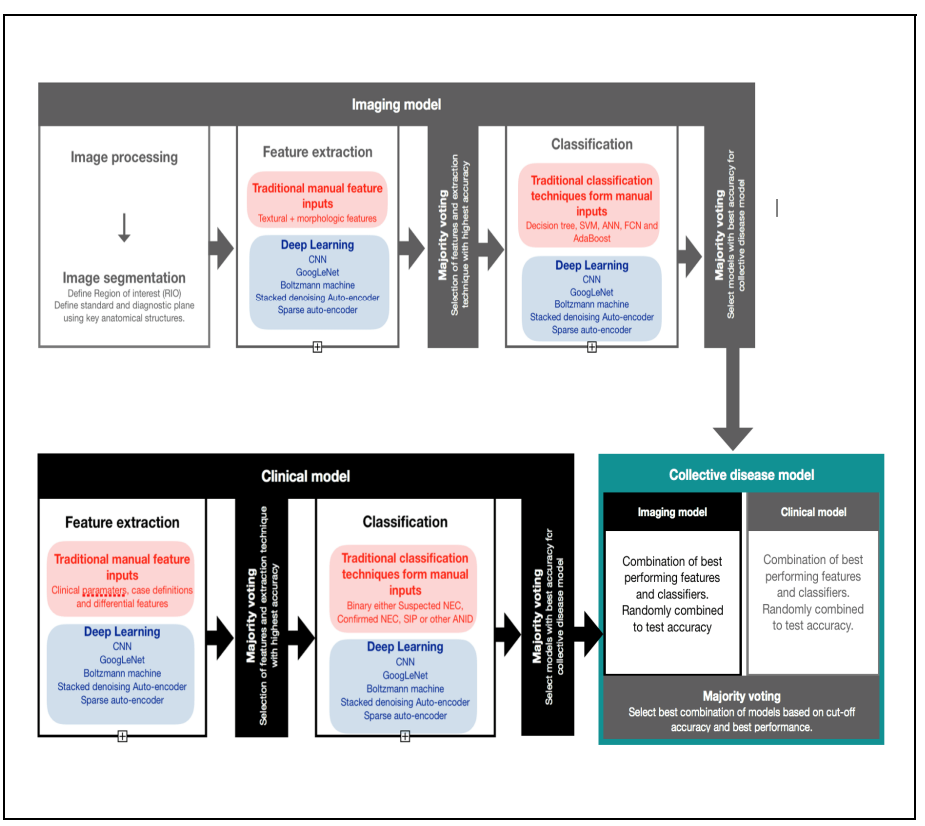

\section{FUTURE RECOMMENDATIONS AND CONCLUSION}

Clinical research as a whole, have a significant challenge ahead. The current set-up costs of once-off trials are causing a trajectory of costs that are unsustainable. Also, as our understanding improves we realise that the complexity of conditions is making it less likely to obtain answers through these traditional trials. A breakthrough in insight into the pathogenesis of some NEC subtypes or NEC mimickers may transform the NEC landscape. However, currently, we do not know if and when that moment will be. Integrating the capabilities of advanced data analytic technologies such as machine learning and CAD yields ongoing insights that can significantly improve clinical practice. The administration, integration, analysis and big data demands of such an understanding will be demanding, but it is an investment that will yield ongoing returns, not only in early diagnosis but continuous evaluation of newer modalities of treatment

The current pace of change and the surmounting challenge to advance not only NEC research but to build case definition of every ANID entity will require a faster pace of adaptation to accommodate personalised medicine. Bedside 
practice in neonatal units needs to be adaptable to embrace new technology to improve productivity, accuracy, and data collection. Predictive modelling holds great promise to advance NEC research and diagnostics. CAD can be the critical enabler to not only save time, resources and cost of research but can also help capture uniform, more productive and dynamic data to improve databases. The big data potential of this is substantial, but careful consideration must be made by neonatal networks to place an international data strategy in place to reap future rewards.

Thorough planning should ensure that the integration of guidelines and regulations in clinical trials design are met. As predictive models are receiving more attention from regulators, failing to meet the requirements will jeopardise efforts. Currently, in Europe, the Standards for Reporting Diagnostic Accuracy Studies (STARD 2015) guidelines define these needs. For multivariate modelling TRIPOD guidelines stipulate standard measures of accuracy as well as transparent reporting for diagnostic model trials. There should be careful consideration of terminology, as defined by FDA BEST, when reporting on the model and their outcomes. As this is a rapidly evolving field, new publications from these regulatory bodies should be carefully monitored for updates.

\section{REFERENCES}

[1] C. Moschopoulos, P. Kratimenos, I. Koutroulis, B. V. Shah, A. Mowes, and V. Bhandari, "The Neurodevelopmental Perspective of Surgical Necrotizing Enterocolitis: The Role of the Gut-Brain Axis," Mediators Inflamm., vol. 2018, p. 7456857, 2018.

[2] S. M. Gephart et al., "Changing the paradigm of defining, detecting, and diagnosing NEC: Perspectives on Bell's stages and biomarkers for NEC," Seminars in Pediatric Surgery, vol. 27, no. 1, pp. 3-10, Feb. 2018.

[3] D. Hackam and M. Caplan, "Necrotizing enterocolitis: Pathophysiology from a historical context," Seminars in Pediatric Surgery, vol. 27, no. 1, pp. 11-18, Feb. 2018.

[4] S. Chen, Y. Hu, Q. Liu, X. Li, H. Wang, and K. Wang, "Comparison of abdominal radiographs and sonography in prognostic prediction of infants with necrotizing enterocolitis," Pediatr. Surg. Int., vol. 34, no. 5, pp. 535-541, May 2018.

[5] "Real-World Data, Real-World Evidence and RealWorld Action | Academy Health." [Online]. Available: http://www.academyhealth.org/blog/201710/real-world-data-real-world-evidence-and-realworld-action. [Accessed: 31-Mar-2018].

[6] "Improving propensity score weighting using machine learning - Lee - 2010 - Statistics in Medicine - Wiley Online Library." [Online]. Available: https://onlinelibrary.wiley.com/doi/full/10.1002/sim.3 782. [Accessed: 31-Mar-2018].

[7] P. Gordon, R. Christensen, J.-H. Weitkamp, and A. Maheshwari, "Mapping the New World of Necrotizing Enterocolitis (NEC): Review and Opinion," EJ Neonatol Res, vol. 2, no. 4, pp. 145172, 2012.
[8] T. Bekhuis and D. Demner-Fushman, "Screening nonrandomized studies for medical systematic reviews: a comparative study of classifiers," Artif Intell Med, vol. 55, no. 3, pp. 197-207, Jul. 2012.

[9] K. B. Cohen et al., "Methodological Issues in Predicting Pediatric Epilepsy Surgery Candidates Through Natural Language Processing and Machine Learning," Biomed Inform Insights, vol. 8, pp. 11-18, May 2016.

[10] C. Han and X. Wei, "Novel biomarkers to determine neonates with necrotizing enterocolitis," Biomed Res, vol. 28 , no. 13 , p. $5,2017$.

[11] Z. Habibi et al., "Predicting ventriculoperitoneal shunt infection in children with hydrocephalus using artificial neural network," Child's Nervous System, vol. 32, no. 11, pp. 2143-2151, 2016.

[12] S. M. Gephart and W. M. Eklund, "NEC Symposium: A transdisciplinary approach to tackle NEC," $A d v$ Neonatal Care, Mar. 2017.

[13] S. M. Gephart, A. R. Spitzer, J. A. Effken, E. Dodd, M. Halpern, and J. M. McGrath, "Discrimination of GutCheck(NEC): a clinical risk index for necrotizing enterocolitis," J Perinatol, vol. 34, no. 6, pp. 468475, Jun. 2014.

[14] S. Hao, S. Geng, L. Fan, J. Chen, Q. Zhang, and L. Li, "Intelligent diagnosis of jaundice with dynamic uncertain causality graph model," Journal of Zhejiang University-SCIENCE B, vol. 18, no. 5, pp. 393-401, May 2017.

[15] J. Barber et al., "A three-dimensional mathematical and computational model of necrotizing enterocolitis," Journal of Theoretical Biology, vol. 322, pp. 17-32, Apr. 2013.

[16] R. Takahashi and Y. Kajikawa, "Computer-aided diagnosis: A survey with bibliometric analysis," International Journal of Medical Informatics, vol. 101, pp. 58-67, May 2017.

[17] H. D. Cheng, J. Shan, W. Ju, Y. Guo, and L. Zhang, "Automated Breast Cancer Detection and Classification Using Ultrasound Images: A Survey," Pattern Recogn., vol. 43, no. 1, pp. 299-317, Jan. 2010.

[18] Q. Huang, F. Zhang, and X. Li, "Machine Learning in Ultrasound Computer-Aided Diagnostic Systems: A Survey," Biomed Res Int, vol. 2018, Mar. 2018.

[19] J. O. Glass, W. E. Reddick, C.-S. Li, F. H. Laningham, K. J. Helton, and C.-H. Pui, "Computeraided detection of therapy-induced leukoencephalopathy in pediatric acute lymphoblastic leukemia patients treated with intravenous high-dose methotrexate," Magnetic Resonance Imaging, vol. 24, no. 6, pp. 785-791, Jul. 2006.

[20] M. Yaqub, B. Kelly, A. T. Papageorghiou, and J. A. Noble, "A Deep Learning Solution for Automatic Fetal Neurosonographic Diagnostic Plane Verification Using Clinical Standard Constraints," Ultrasound Med Biol, vol. 43, no. 12, pp. 2925-2933, Dec. 2017.

[21] R. Faingold et al., "Necrotizing enterocolitis: assessment of bowel viability with color doppler US," Radiology, vol. 235, no. 2, pp. 587-594, May 2005.

[22] M. Epelman et al., "Necrotizing enterocolitis: review of state-of-the-art imaging findings with pathologic 
correlation," Radiographics, vol. 27, no. 2, pp. 285305, Apr. 2007.

[23] K. Muchantef, M. Epelman, K. Darge, H. Kirpalani, P. Laje, and S. A. Anupindi, "Sonographic and radiographic imaging features of the neonate with necrotizing enterocolitis: correlating findings with outcomes," Pediatr Radiol, vol. 43, no. 11, pp. 1444 1452, Nov. 2013.

[24] B. Bohnhorst, "Usefulness of abdominal ultrasound in diagnosing necrotising enterocolitis," Archives of Disease in Childhood - Fetal and Neonatal Edition, vol. 98, no. 5, pp. F445-F450, Sep. 2013.

[25] A. Fischer, L. Vachon, M. Durand, and R. G. Cayabyab, "Ultrasound to diagnose spontaneous intestinal perforation in infants weighing $\leqslant 1000 \mathrm{~g}$ at birth," J Perinatol, vol. 35, no. 2, pp. 104-109, Feb. 2015.

[26] A. C. Cuna, J. C. Lee, A. L. Robinson, N. H. Allen, J. E. Foley, and S. S. Chan, "Bowel Ultrasound for the Diagnosis of Necrotizing Enterocolitis: A Metaanalysis," Ultrasound Q, Jan. 2018.

[27] A. C. Cuna, N. Reddy, A. L. Robinson, and S. S. Chan, "Bowel ultrasound for predicting surgical management of necrotizing enterocolitis: a systematic review and meta-analysis," Pediatr Radiol, vol. 48, no. 5, pp. 658-666, May 2018.

[28] D. Ni et al., "Standard Plane Localization in Ultrasound by Radial Component Model and Selective Search," Ultrasound in Medicine \& Biology, vol. 40, no. 11, pp. 2728-2742, Nov. 2014.

[29] J. Chi, E. Walia, P. Babyn, J. Wang, G. Groot, and M. Eramian, "Thyroid Nodule Classification in Ultrasound Images by Fine-Tuning Deep Convolutional Neural Network," J Digit Imaging, vol. 30, no. 4, pp. 477-486, Aug. 2017.

[30] S. Han et al., "A deep learning framework for supporting the classification of breast lesions in ultrasound images," Phys. Med. Biol., vol. 62, no. 19, p. 7714, 2017.

[31] Y. Yu, Y. Xiao, J. Cheng, and B. Chiu, "Breast lesion classification based on supersonic shear-wave elastography and automated lesion segmentation from B-mode ultrasound images," Computers in Biology and Medicine, vol. 93, pp. 31-46, Feb. 2018.
[32] J. Ma, F. Wu, T. Jiang, J. Zhu, and D. Kong, "Cascade convolutional neural networks for automatic detection of thyroid nodules in ultrasound images," Medical Physics, vol. 44, no. 5, pp. 1678-1691.

[33] X. Liu, J. L. Song, S. H. Wang, J. W. Zhao, and Y. Q. Chen, "Learning to Diagnose Cirrhosis with Liver Capsule Guided Ultrasound Image Classification," Sensors (Basel), vol. 17, no. 1, Jan. 2017.

[34] T. M. Hassan, M. Elmogy, and E.-S. Sallam, "Diagnosis of Focal Liver Diseases Based on Deep Learning Technique for Ultrasound Images," Arabian Journal for Science and Engineering, vol. 42, no. 8, pp. 3127-3140, Aug. 2017.

[35] J.-Z. Cheng et al., "Computer-Aided Diagnosis with Deep Learning Architecture: Applications to Breast Lesions in US Images and Pulmonary Nodules in CT Scans," Scientific Reports, vol. 6, no. 1, Jul. 2016.

[36] Y.-L. Huang, D.-R. Chen, Y.-R. Jiang, S.-J. Kuo, H.$\mathrm{K}$. Wu, and W. K. Moon, "Computer-aided diagnosis using morphological features for classifying breast lesions on ultrasound," Ultrasound in Obstetrics \& Gynecology, vol. 32, no. 4, pp. 565-572.

[37] D. Meng, L. Zhang, G. Cao, W. Cao, G. Zhang, and B. Hu, "Liver Fibrosis Classification Based on Transfer Learning and FCNet for Ultrasound Images," IEEE Access, vol. 5, pp. 5804-5810, 2017.

[38] U. R. Acharya et al., "Thyroid lesion classification in 242 patient population using Gabor transform features from high resolution ultrasound images," KnowledgeBased Systems, vol. 107, pp. 235-245, Sep. 2016.

[39] U. R. Acharya et al., "An integrated index for identification of fatty liver disease using radon transform and discrete cosine transform features in ultrasound images," Information Fusion, vol. 31, pp. 43-53, Sep. 2016.

[40] U. R. Acharya, O. Faust, S. V. Sree, F. Molinari, and J. S. Suri, "ThyroScreen system: high resolution ultrasound thyroid image characterization into benign and malignant classes using novel combination of texture and discrete wavelet transform," Comput Methods Programs Biomed, vol. 107, no. 2, pp. 233241, Aug. 2012. 